\title{
Spin-Based Quantum Dot Qubits
}

\author{
V. N. Stavrou ${ }^{1}$ and G. P. Veropoulos 2 \\ ${ }^{1}$ Department of Physics and Astronomy, University of Iowa, Iowa City \\ ${ }^{2}$ Division of Physics, Hellenic Naval Academy, Hadjikyriakou, \\ and Division of Academic Studies, Hellenic Navy Petty Officers Academy, Skaramagkas, \\ 1 USA \\ ${ }^{2}$ Greece
}

\section{Introduction}

Spin-based electronics in low dimensional structure (LDS) e.g. quantum wells (QWs) and quantum dots (QDs) (especially in QDs) have recently attracted a lot of interest for applications in quantum information technology (Burkard et al., 1999; Imamoglu et al., 1999; Nielsen \& Chuang, 2000). Two-dimensional (2-d) QDs (Baruffa et al., 2010; Bertoni et al., 2005; Fessatidis et al., 1999; Foulkes et al., 2001; Hayashi et al., 2004; Hu \& Sarma, 2000; 2001; Räsänen et al., 2003; Simserides et al., 2000; 2006; 2007; Stano \& Fabian, 2005; 2006; Thorwart et al., 2005; Witzel et al., 2007) and self-assembled QDs (SAQDs) (Artús et al., 2000; Bányai \& Koch, 1993; Fujisawa et al., 1998; 2006; Lee et al., 1998; 2004; Pryor et al., 1997; Pryor, 1998; Pryor \& Flatté, 2003) among other heterostructures have been suggested for constructing quantum bits (qubits). Quantum computing architecture mainly uses the properties of QDs related to charge and spin of the confined carriers, among others. Thus, the carrier relaxation via the emission of phonons (Khaetskii \& Nazarov, 2001; Mohanty, 2000; Stavrou \& Hu, 2005; 2006; Stavrou, 2007; Woods et al., 2002) and the light polarization interplay with the spin-polarized states within QDs (Chye et al., 2002; Pryor \& Flatté, 2003; Stavrou, 2008; 2009), which are electrically injected into QDs, are of special importance in quantum computing technology.

During the last decade, a few theoretical and experimental reports have been announced related to light polarization within self-assembled quantum dots (Cantele et al., 2001; 2002; Chye et al., 2002; Pryor \& Flatté, 2003; Stavrou, 2008; 2009). More specifically, they have studied circular polarization dependence of dipole recombination of spin-polarized states within a self-assembled quantum dots. The comparison between the theoretical estimation (Pryor \& Flatté, 2003) and the experimental report (Chye et al., 2002) shows that the theoretical model can successfully describe the experiment.

In this chapter, we review the circularly polarized light along the orientation of spin-polarized carriers in a system of two coupled SAQDs made with InAs/GaAs. The dependence of circular light polarization on the geomerty parameters, the absence of an external magnetic field and the presence of a magnetic field is the main part of our investigation We can highlight the most important results along the direction [110]: a) for large interdot distances (uncoupled single QDs), our results are consistent with the polarization along to the direction [110], of the emitted light for the case of a single QD ( 5\%, Ref. (Chye et al., 2002; Pryor \& Flatté, 


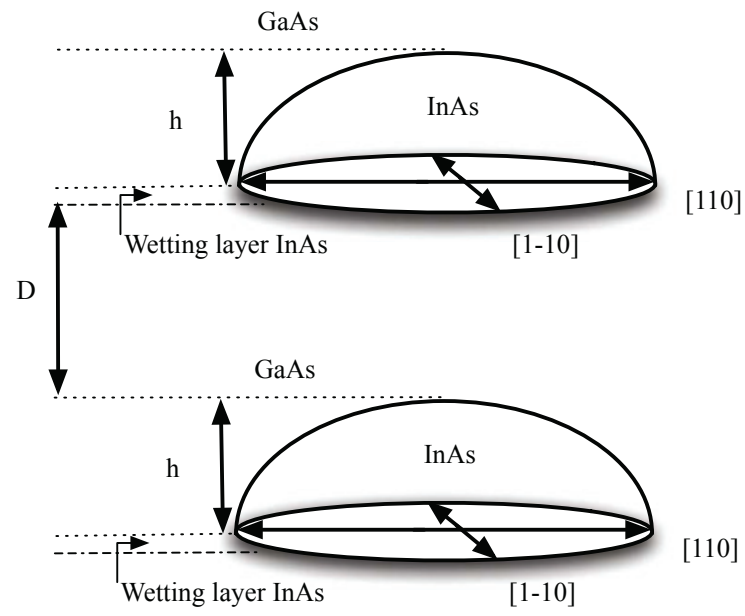

Fig. 1. The Geometry of the coupled ellipsoidal-shaped quantum dot heterostructure made with InAs/GaAs and a fixed wetting layer thickness to $0.3 \mathrm{~nm}$. The two quantum dots are identical and the dot height is fixed to $h=2.1 \mathrm{~nm}$. The thickness of the wetting layer must be included in the height of the QDs in order to achieve the comparison to the experimental results.

2003) ), b) for smaller distances the polarization increases receiving maximum value for our typical geometry $\sim 40 \%$, c) in the case of large QDs the polarization decreases by increasing the magnetic field and for small dots the polarization increases, d) circular polarization along other polarization directions shows that light polarization does not depend on the above mentioned parameters.

This chapter is arranged as follows: In section 2, we briefly review the most recent SAQS fabrication methods. The eight-band $\mathbf{k} \cdot \mathbf{p}$ theory and light polarization theory are described in section 3 . In section 4 , we present and comment the numerical results. Lastly, the conclusions are placed in section 5 .

\section{Self-assembled QDs}

SAQDs are nanostructures which have been worldwide used in nanotechnology e.g. quantum computing devices. The fabrication techniques of the SACQDs are not a simple formation mechanism but appears to be difficult and not easy to be controlled (Barabási \& Stanley, 1995; Barabási, 1997; Darula \& Barabási, 1997; Daudin et al., 1997; Dawson et al., 2007; Eaglesham \& Cerullo, 1990; Lee et al., 1998; 2004; Rastelli et al., 2005; Tersoff, 1995; 1998; Tu \& Tersoff, 2007). The various parameters (e.g. thermodynamic parameters) which influence the SACQDs growth are not well understood and as a result the shape of the SACQDs, which are very important in determining confining energies of electrons/holes, can not be easily achieved with high quality. Semiconductor QDs growth techniques which have been often used are the colloidal methods (Blackburn et al., 2003; Chestnoy et al., 1986; Wang \& Herron, 1991) 


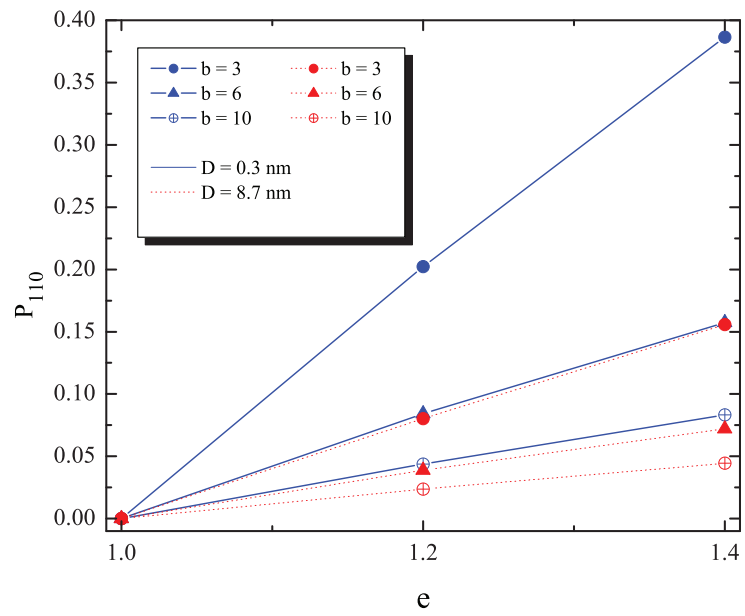

Fig. 2. The circular light polarization along the direction [110] dependence on the elongation for different separation distances, width-to-height ratio and fixed dot height $h=2.1 \mathrm{~nm}$. There is not an applied external magnetic field to the structure.

and the high-resolution electron lithography. (Lebens et al., 1990; Owen, 1985; Temkin et al., 1987) The main problem of these growth techniques is the incorporation of the defects. In fabricating SAQDs, these difficulties were overcome by using the Stranski-Krastanov (SK) coherent island growth mode. SK growth is one of the three primary modes by which thin films grow epitaxially at crystal surfaces/interfaces.

Theoretical and computational studies in epitaxial crystal growth is highly attractive in QDs sample fabrication. Several research groups have studied several growth parameters which play a crucial role during the self-organized growth of semiconductor quantum dot structures in the Stranski-Krastanov growth mode. Among others, the strain field of the lattice-mismatched systems, the growth temperature V/III ratio and the QD growth time are a few parameters which are of crucial importance in theoretical studies and fabrication technology. Kinetic Monte-Carlo simulations (Biehl \& Much, 2003; 2005; Ghaisas \& Madhukar, 1986; Kew et al., 1993; Khor \& Sarma, 2000; Lam et al., 2002; Madhukar, 1983; Much et al, 2001; Ohr et al, 1992; Sitter, 1992) have been used to describe the wetting-layer and the island formation in heteroepitaxial growth.

\section{Theory}

Computational algorithms which have been used to solve Schrodinger equation in nanostructures are density functional theory (DFT) (Gross et al., 1991; Hohenberg \& Kohn, 1964; Kohn \& Sham, 1965; Sham \& Kohn, 1966), Monte Carlo techniques (Foulkes et al., 2001; Räsänen et al., 2003; Thijssen, 1998), direct diagonalization techniques (Sadiku, 2001; Thijssen, 1998; Varga et al., 2011) and k · p theory (Bahder, 1990; 1992; Bimberg et al., 1999; 


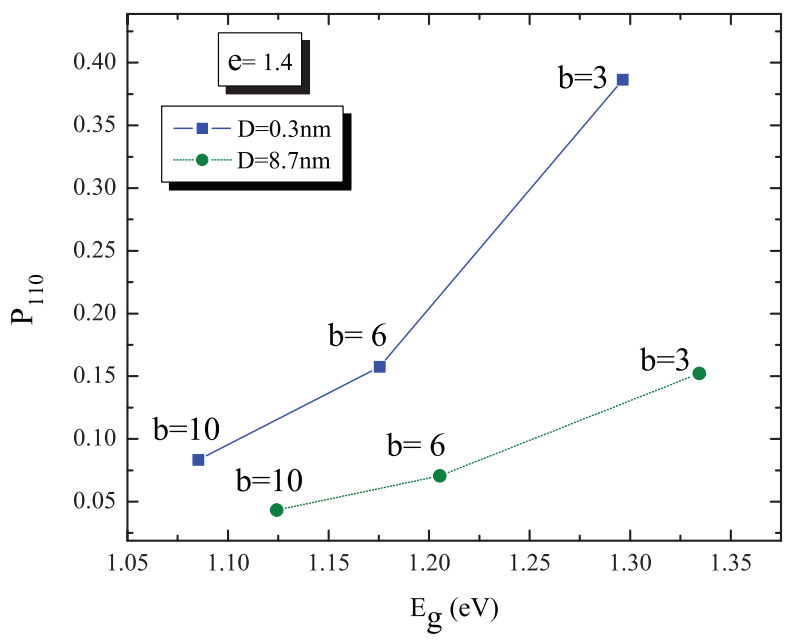

Fig. 3. The circular light polarization along the direction [110] as a function of the energy gap for different interdot distances and width-to-height ratio with fixed dot height $h=2.1 \mathrm{~nm}$ and elongation $e=1.4$ in the absence of an external magnetic field.

Gershoni et al., 1993; Pryor et al., 1997; Pryor, 1998), among others. Although, k · p is used to describe single electron/hole wavefunctions in heterostructures, all the other above mentioned algorithms can also solve Schrodinger equation in heterostructures with a large number of electrons/holes.

In this section, we theoretically describe the $\mathbf{k} \cdot \mathbf{p}$ model for the case of single electron/hole quantum dots. The evaluation of wavefunctions will be used in our present research concerning the circular light polarization. The 8 -band $\mathbf{k} \cdot \mathbf{p}$ model requires a basis of eight Bloch functions. The wavefunctions related to the Bloch functions are the following

$$
\left\{\psi_{s, \uparrow}, \psi_{x, \uparrow}, \psi_{y, \uparrow}, \psi_{z, \uparrow}, \psi_{s, \downarrow}, \psi_{x, \downarrow}, \psi_{y, \downarrow}, \psi_{z, \downarrow}\right\}
$$

where $x, y, y$ are related to three directions of the space and the arrows denote the spin.

The 8-band k · p Hamiltonian (Bahder, 1990; 1992; Bimberg et al., 1999) is described by the following $2 \times 2$ block matrix form

$$
\hat{\mathcal{H}}=\left(\begin{array}{cc}
G(\mathbf{k}) & \Gamma \\
-\bar{\Gamma} & \bar{G}(-\mathbf{k})
\end{array}\right)
$$

where $\Gamma$ and $\mathrm{G}$ are $4 x 4$ matrices. The matrix $\Gamma$ couples the spin projections $\uparrow$ and $\downarrow$ due to spin-orbit (SO) interaction and matrix G consists of the potential energy, the kinetic energy, a $\mathrm{SO}$ interactions and a strain dependent part. 


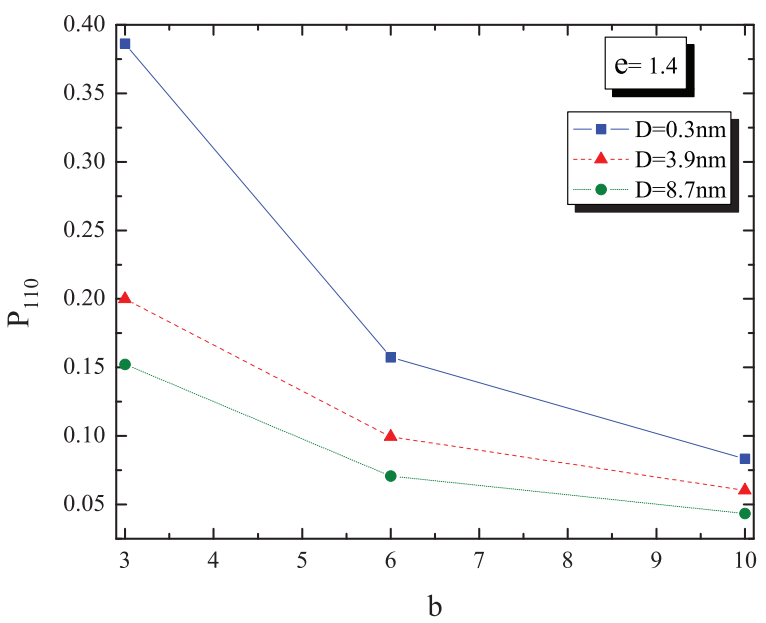

Fig. 4. The circular light polarization along the direction [110] as a function of width-to-height ratio with fixed elongation and different interdot distances with fixed dot height $h=2.1 \mathrm{~nm}$ in the absence of an external magnetic field.

$$
\Gamma=\left(\begin{array}{cccc}
0 & 0 & 0 & 0 \\
0 & 0 & 0 & \Delta_{0} / 3 \\
0 & 0 & 0 & -i \Delta_{0} / 3 \\
0 & -\Delta_{0} / 3 & i \Delta_{0} / 3 & 0
\end{array}\right)
$$

where $\Delta_{0}$ is the splitting between the $\Gamma_{8}$ and $\Gamma_{7}$ valence bands (Bahder, 1990; 1992; Bimberg et al., 1999).

The matrix $G$ is given by the summation of a potential energy part $\left(G_{1}\right)$, a kinetic energy part $\left(G_{2}\right)$, a SO interaction part $\left(G_{S O}\right)$ and a strain dependent part $\left(G_{s t}\right)$

$$
G=G_{1}+G_{2}+G_{S O}+G_{s t}
$$

where the matrices $G_{1}, G_{2}, G_{S O}$ and $G_{s t}$ are respectively given by

$$
\begin{gathered}
G_{1}=\left(\begin{array}{cccc}
E_{c} & i P k_{x} & i P k_{y} & i P k_{z} \\
-i P k_{x} & E_{v}^{\prime} & 0 & 0 \\
-i P k_{y} & 0 & E_{v}^{\prime} & 0 \\
-i P k_{z} & 0 & 0 & E_{v}^{\prime}
\end{array}\right) \\
G_{2}=\left(\begin{array}{cccc}
A^{\prime} k^{2} & B k_{y} k_{z} & B k_{x} k_{z} & B k_{x} k_{y} \\
B k_{y} k_{z} & L^{\prime} k_{x}^{2}+M\left(k_{y}^{2}+k_{z}^{2}\right) & N^{\prime} k_{x} k_{y} & N^{\prime} k_{x} k_{z} \\
B k_{z} k_{x} & N^{\prime} k_{x} k_{y} & L^{\prime} k_{y}^{2}+M\left(k_{x}^{2}+k_{z}^{2}\right) & N^{\prime} k_{y} k_{z} \\
B k_{x} k_{y} & N^{\prime} k_{x} k_{z} & N^{\prime} k_{y} k_{z} & L^{\prime} k_{z}^{2}+M\left(k_{x}^{2}+k_{y}^{2}\right)
\end{array}\right)
\end{gathered}
$$



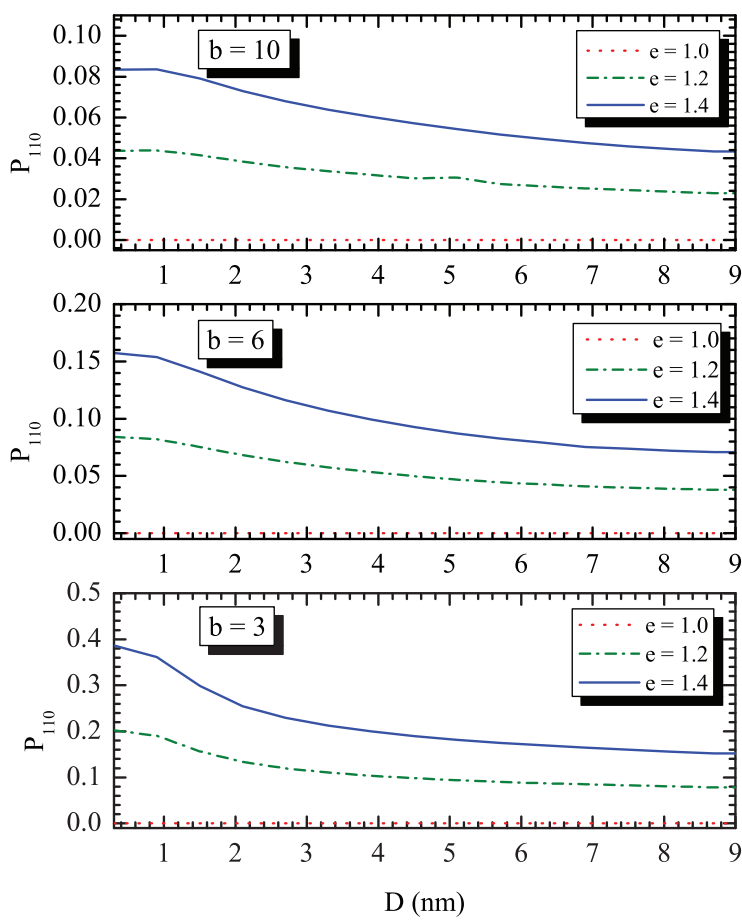

Fig. 5. The circular light polarization along the direction [110] as a function of the interdot distance for different elongation and width-to-height ratio with fixed dot height $h=2.1 \mathrm{~nm}$. There is not an applied external magnetic field to the structure.

$$
G_{S O}=\left(\begin{array}{cccr}
0 & 0 & 0 & 0 \\
0 & 0 & -i \Delta_{0} / 3 & 0 \\
0 & -i \Delta_{0} / 3 & 0 & 0 \\
0 & 0 & 0 & 0
\end{array}\right)
$$

and

$$
G_{s t}=\left(\begin{array}{cccc}
a_{c}\left(\epsilon_{x x}+\epsilon_{y y}+\epsilon_{z z}\right) & b^{\prime} \epsilon_{y z}-i P \epsilon_{x j} k^{j} & b^{\prime} \epsilon_{z x}-i P \epsilon_{y j} k^{j} & b^{\prime} \epsilon_{x y}-i P \epsilon_{z j} k^{j} \\
b^{\prime} \epsilon_{y z}+i P \epsilon_{x j} k^{j} & l \epsilon_{x x}+m\left(\epsilon_{y y}+\epsilon_{z z}\right) & n \epsilon_{x y} & n \epsilon_{x z} \\
b^{\prime} \epsilon_{z x}+i P \epsilon_{y j} k^{j} & n \epsilon_{x y} & l \epsilon_{y y}+m\left(\epsilon_{x x}+\epsilon_{z z}\right) & n \epsilon_{y z} \\
b^{\prime} \epsilon_{x y}+i P \epsilon_{z j} k^{j} & n \epsilon_{x z} & n \epsilon_{y z} & l \epsilon_{x x}+m\left(\epsilon_{y y}+\epsilon_{z z}\right)
\end{array}\right)
$$

where all the parameters used within the matrices are given by Refs (Bimberg et al., 1999; Gershoni et al., 1993; Saada, 1989). 


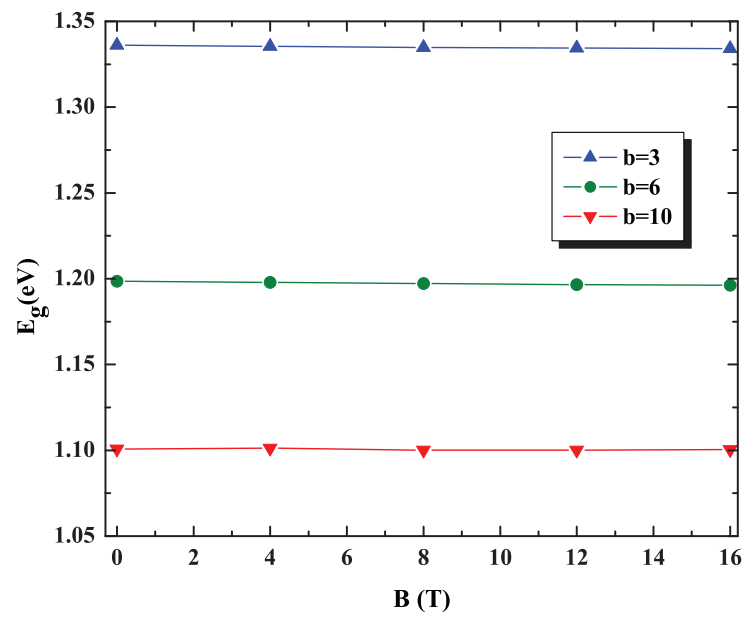

Fig. 6. The energy gap as a function of the applied magnetic field for different width-to-height ratio and fixed elongation $(\mathrm{e}=1.4)$.

In our study, we have considered two ellipsoidal caps QDs made with InAs which are separated by a distance $D$ as illustrated in Fig.1. The QDs are embedded on a wetting layer of InAs and are surrounded by GaAs. Single electron and hole wavefunctions are calculated using the strain dependent $\mathbf{k} \cdot \mathbf{p}$ theory. In our calculations, we have fixed the height of the dots to $h=2.1 \mathrm{~nm}$ and varied the width-to-height ratio

$$
b=\left(d_{[110]}+d_{[1-10]}\right) / h
$$

and elongation

$$
e=d_{[110]} / d_{[1-10]}
$$

The electron/hole wavefunctions were numerically computed on a real space grid with spacing equal to the wetting layer thickness $0.3 \mathrm{~nm}$. Strain and carrier confinement split the heavy hole $(\mathrm{HH})$ and light hole ( $\mathrm{LH})$ degeneracy and the states are doubly degenerate which are denoted by $\mid \psi>$ and $T \mid \psi>$ (time reverses of each other). The energy gap $\left(E_{g}\right)$ of the coupled SAQD structure strongly depends on the interdot distance as it is shown in our investigation. All material parameters that have been used in the $\mathbf{k} \cdot \mathbf{p}$ simulations are taken by Ref. (Pryor1, Vurgaftman). Spin polarized ground states are constructed by taking a linear combination of the states comprising the doublet and adjusting the coefficient in order to maximize the expectation value of the pseudospin operator projected onto a direction $l$ (Pryor $\&$ Flatté, 2003). The requested complex number $\alpha$ maximizes the following

$$
\frac{\left[<\psi\left|+\alpha^{*}<\psi\right| T\right] \hat{l} \cdot \mathbf{S}[|\psi>+\alpha T| \psi>]}{1+|\alpha|^{2}}
$$



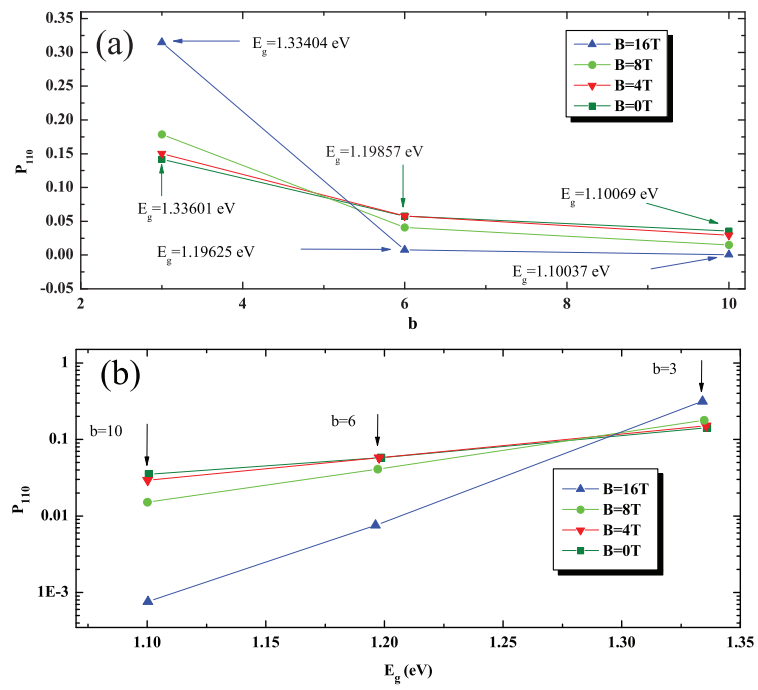

Fig. 7. (a) The circular light polarization along the direction [110] as a function of the width-to-height ratio for different applied magnetic fields with fixed dot height $h=2.1 \mathrm{~nm}$ and elongation $e=1.4$. (b) The circular light polarization along the direction [110] as a function of the energy gap for different applied magnetic fields with fixed dot height $h=2.1 \mathrm{~nm}$ and elongation $e=1.4$.

where $\mathbf{S}$ is the pseudospin operator (Pryor et al., 1997; Pryor, 1998; Pryor \& Flatté, 2003) in the 8 -band $\mathbf{k} \cdot \mathbf{p}$ theory

$$
\overrightarrow{\mathbf{S}}=\left(\begin{array}{ccc}
\vec{\sigma} \Gamma_{6} & 0 & 0 \\
0 & \vec{J} \Gamma_{8} & 0 \\
0 & 0 & \vec{\sigma} \Gamma_{7}
\end{array}\right)
$$

and $l$ the spin orientation.

Let us consider the situation in which the electron spin is polarized along the same direction $l$ as the observed emitted light. The polarization which characterizes the emitted light is given by (Jackson, 1998)

$$
P_{l}=\frac{I_{l}^{(+)}-I_{l}^{(-)}}{I_{l}^{(+)}+I_{l}^{(-)}}
$$

where $I_{l}^{( \pm)}$is the light intensity with \pm helicity. The intensity of emission of circularly polarized light for the case of spin-polarized electron and unpolarized holes is given by

$$
I_{l}^{( \pm)}=\mid\left\langle\psi_{h}\left|\hat{\epsilon}_{l}^{( \pm)} \cdot \mathbf{p}\right| \psi_{e}>\left.\right|^{2}+\right|\left\langle\psi_{h}\left|T \hat{\epsilon}_{l}^{( \pm)} \cdot \mathbf{p}\right| \psi_{e}>\left.\right|^{2}\right.
$$



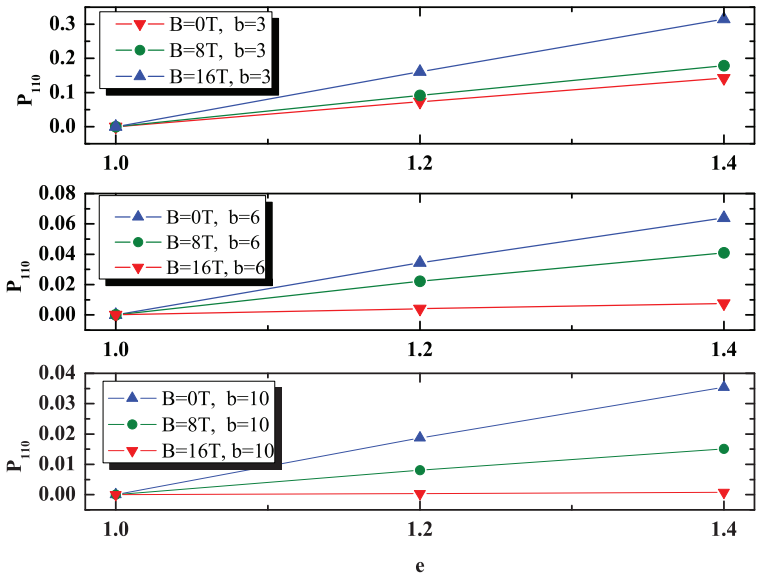

Fig. 8. The circular light polarization along the direction [110] as a function of the elongation for different applied magnetic fields and width-to-height ratio with fixed dot height $h=2.1 \mathrm{~nm}$.

and for the case of spin-polarized holes and unpolarized electron the intensity of circularly polarized light is given by

$$
I_{l}^{( \pm)}=\mid\left\langle\psi_{h}\left|\hat{\epsilon}_{l}^{( \pm)} \cdot \mathbf{p}\right| \psi_{e}>\left.\right|^{2}+\right|\left\langle\psi_{h}\left|\hat{\epsilon}_{l}^{( \pm)} \cdot \mathbf{p} T\right| \psi_{e}>\left.\right|^{2}\right.
$$

where the indices $e$ and $h$ correspond to electron and holes respectively, $\mathbf{p}$ is the momentum operator and $\hat{\epsilon}_{l}^{( \pm)}$is the circular polarization vector with helicity \pm (which denotes circularly polarized light that propagates along direction $l$ ). It is worth mentioning that the last two equations give identical results as a result of the anticommutation relations between $p$ and $T$. As a result the emitted light polarization is independent, no matter if the injected spin-polarized carriers are electrons or holes.

\section{Results}

In this work, we research the circular light polarization along the plane [110] (as illustrated in Fig.1) for $100 \%$ spin-polarized carriers along [110]. The dependence of light polarization on the elongation is shown at Fig.2 for different interdot distances and QD sizes (width-to-height ratio). For axially symmetric QDs the polarization is zero because the intensities with different helicity are equal and in the case of more elongated QDs the light polarization increases due to the azimuthal symmetry breaking. Furthermore, circular light polarization increases as QDs come close to each other and as the size of the dots decreases. This happens as a result of the wavefunction dependence on the QDs geometry. Increasing the interdot distance the electron energies in two lowest conduction band converge towards that of an electron in a single QD. The energy difference between the ground state and first excited state decreases as the distance increases. On the other hand, in the valence band the energy splitting decreases as 

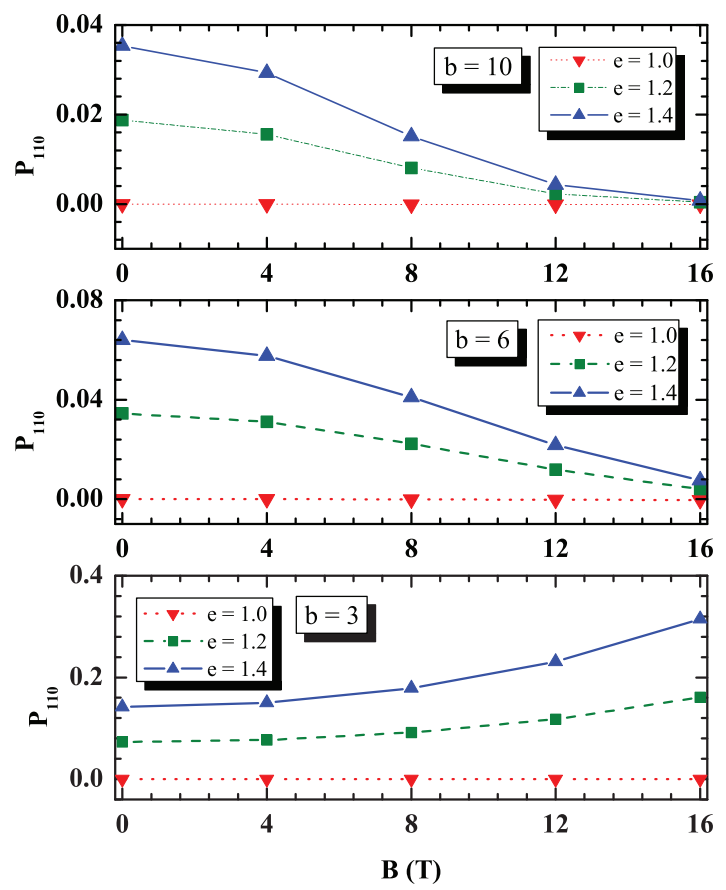

Fig. 9. The circular light polarization along the direction [110] as a function of the applied magnetic field for different elongation and width-to-height ratio with fixed dot height $h=2.1 \mathrm{~nm}$.

the separation distance increases. Both conduction and valence band ground energies increase by increasing the separation distance (Bimberg et al., 1999; Stavrou, 2008). As a result the energy gap increases by increasing the interdot distance. Fig. 3, presents the polarization as a function of energy gap for different geometric parameters. It is clear that increasing the width-to-height ratio light polarization decays and increases as the separation decreases because of the wavefunction dependence on the geometric parameters. In the case of large elongation $(e=1.4)$, small dot size $(b=3)$ and interdot distance $(D=0.3 \mathrm{~nm})$ the polarization has the largest value ( $P_{110}=0.38$, the emitted light is $38 \%$ polarized). Polarization has the smallest values for large dots for all the interdot distances as it is illustrated in Fig.3. The dependence of circular light polarization on the width-to-height ratio is presented in Fig.4. For small separation distance the polarization becomes large and as the separation distance increases the light polarization decreases.

A complete picture of the dependence of circular light polarization on all the above mentioned parameters is illustrated in Fig.5. The light polarization efficiency along the direction [110] strongly depends on the interdot distance, the size and the elongation of the QD. In the limit of 
large interdot distance (uncoupled QDs) and elongation $e=1.4$ the results are consistent with the numerical calculations previously reported (Pryor \& Flatté, 2003) and the experimental results (Chye et al., 2002) ( 5\% circularly polarized light for 100\% polarized carriers). For other polarization directions like [001] the polarization efficiency it founds to be very close to $1\left(P_{001}=0.99\right)$ and almost independent on the interdot distance. So $\left(P_{001}=0.99\right)$ is the best choice if one is interested in the light polarization along the growth direction. Along the directions [100] and [010] the circular polarization vanishes $P_{100}=P_{010}=0$ for any of our QD geometry.

The next part of our investigation is related to circular light polarization dependence on an applied external magnetic field. We have considered the case of a single QD in the presence of a magnetic field. The variation of the magnetic field releases the dependence on the Zeeman energy. As the magnetic field increases the energy splitting increases due to the Zeeman energy splitting. The dependence of the energy gap on the magnetic field is presented in Fig.6 for different sizes of QDs. The current investigation shows that the energy gap strongly depends on the size of the dot and less on the external magnetic field (Fig.6). In the case of small dots $\left(b=3\right.$ ) and $B=0 T$ (or $B=16 T$ ) the energy gap gets the value $E_{g}=1.336 \mathrm{eV}$ (or $E_{g}=1.334 \mathrm{eV}$ ). Therefore, for the above case, the energy gap difference is $0.002 \mathrm{eV}$ which is a tiny difference. The existence of the magnetic field does not change the energy difference $\left(E_{g}\right)$ between the ground states in conduction band and valence band. Thus, the size of the dots is the dominant parameter that changes drastically the energy gap due to the fact that for the case of small QDs the electrons (holes) have larger energy than in the case of larger QDs which results in larger energy difference between the carrier in the bottom of the conduction and the top valence band state.

The dependence of circular polarization on the size and on the energy gap is given in Fig.7. In the case of fixed width-to-height ratio $\mathrm{b}=3$, the smallest value of $P_{110}$ is achieved for the case of $\mathrm{B}=0 \mathrm{~T}$ and the largest for $\mathrm{B}=16 \mathrm{~T}$, although the energy gap slightly differs. Fig.7(b) presents the dependence of circular light polarization on the QD energy gap. As we have earlier shown (absence of magnetic field), in the case of the presence of magnetic field and $e=1.0$ (axial symmetric QD), light polarization vanishes because of unchanging value of light intensity for \pm helicity (Fig.8). Increasing the elongation, circular light polarization increases because of the azimuthal symmetry breaking. Light polarization increases as the magnetic field increases and decreases as the size of the quantum dots increases, as earlier explained.

The light polarization as a function of the external magnetic field, the separation distance, the size of the dots and the elongation is presented in Fig.9. As it is obvious, in small (large dots), polarizarion increases (decreases) by increasing the magnetic field. Responsible for this kind of behavior is the dependence of the energy gap on the size of QD and the Zeeman splitting due to the external magnetic field (Stavrou, 2009). As earlier mentioned, the energy gap of small dots is larger than that of large dots. Furthermore, the carrier wavefunctions involved in Eq. (13) depend on the size of the QDs and result in larger polarization for the case of small dots (large $E_{g}$ ). Increasing the magnetic field in small QDs, the light polarization gets larger values as a consequence of carrier wavefunctions dependence on the magnetic field. Although the carriers are $100 \%$ polarized, the emitted light is less than $100 \%$ polarized. In the case of $B=0 \mathrm{~T}$ and elongation $e=1.4$ the results are consistent with the numerical calculations (Pryor \& Flatté, 2003) and the experimental results (Chye et al., 2002) ( 5\% circularly polarized light for $100 \%$ polarized carriers) previously reported. Increasing the $E_{g}$, it is possible for small QDs to increase the percentage of polarized light by increasing the external magnetic field. 
For small QDs, $B=0 T$ and $e=1.4$ the light is $\sim 18 \%$ polarized and for $B=16 \mathrm{~T}, e=1.4$ the light is $\sim 32 \%$ polarized. The dependence of the light polarization on the separation distance in the presence of an external field is under investigation by our group.

Lastly, we mention that for other polarization directions other than [110], the light polarization has different behavior, as in the case of the absence of the the magnetic field. The circular polarization along the directions [100], [010] is zero and 1 along the direction [001] and appears to be independent on magnetic field.

\section{Conclusions}

Summing overall, we have researched a possible qubit made with SAQDs. Our investigation shows that for the case of absence of an external magnetic field, although the carriers are $100 \%$ polarized the emitted light is less than $100 \%$ polarized. The largest polarization $\left(P_{110}=0.38\right)$ was generated for small $\mathrm{D}, \mathrm{b}=3$ and $\mathrm{e}=1.4$. Thus, the observed $1 \%$ polarization was generated by carriers that were $1 / 0.38 \sim 2.6 \%$ polarized. The circular light polarization depends strongly on the interdot distance (Fig.5), the size of the dots and the elongation. On the other hand, in the presence of an external magnetic field, it has been shown that both the magnetic field and the geometry of the QD are parameters of special importance in order to control the light polarization along the direction of spin polarization in QD qubits (Fig.9). For small dots, circular light polarization along the direction [110] increases by increasing the magnetic field and in the case of large dots it decreases as the field increases. Lastly, other polarization directions like [001] and [100] lead to circular light polarization value 1 and 0 respectively.

\section{Acknowledgment}

The authors would like to thank Prof. C. Pryor, for useful discussions and Commander of the Hellenic Navy D. Filinis for his support. The author V.N.S. would like also to acknowledge the financial support given by the Training Mobility of Researchers (TMR), NSA and ARDA under ARO Contract No. DAAD19-03-1-0128. and the University of Iowa under the grand No. 52570034 .

\section{References}

Artús, L., Cuscó, R., Hernández, S., Patanè, A., Polimeni, A., Henini, M. \& Eaves, L. (2000). Quantum-dot phonons in self-assembled InAs/GaAs quantum dots: Dependence on the coverage thickness, Appl. Phys. Lett., 77, (3556-3558).

Bahder, T.,B. (1990). Eight-band k.p model of strained zinc-blende crystals, Phys. Rev. B 41, (11992-12001).

Bahder, T.,B. (1992). Analytic dispersion relations near the $\Gamma$ point in strained zinc-blende crystals, Phys. Rev. B 45, (1629-1637).

Bányai, L. \& Koch, S.W. (1993). Semiconductor Quantum Dots (World Scientific).

Barabási, A.,L \& Stanley, H.,E. (1995). Fractal Concepts in Surface Growth, (Cambridge University Press, Cambridge).

Barabási, A.,L, (1997). Self-assembled island formation in heteroepitaxial growth, Appl. Phys. Lett., 70, (2565-2567).

Baruffa, F., Stano, P. \& Fabian, J. (2010). Theory of Anisotropic Exchange in Laterally Coupled Quantum Dots, Phys. Rev. Lett, 104, (126401-126401). 
Bertoni, A., Rontani, M., Goldoni, G. \& Molinari, E. (2005). Reduced Electron Relaxation Rate in Multielectron Quantum Dots, Phys. Rev. Lett., 95, (066806-066809)

Biehl, M. \& Much, F. (2003). Simulation of wetting-layer and island formation in heteroepitaxial growth, Europhys. Lett., 63,(14-20).

Biehl, M. \& Much, F. (2005). Off-lattice Kinetic Monte Carlo simulations of Stranski-Krastanov-like growth, B. A. Joyce et al. (eds.), Quantum Dots: Fundamentals, Applications, and Frontiers, (89-102) (Springer).

Blackburn, J.,L., Ellingson, R.J., Micic, O.,I. \& Nozik, A.,J. (2003) Electron Relaxation in Colloidal InP Quantum Dots with Photogenerated Excitons or Chemically Injected Electrons, J. Phys. Chem. B 107, (102-109).

Bimberg, D., Grundmann, M. \& Ledentsov, N.,N. (1999). Quantum Dot Heterostructures (Wiley-VCH Verlag, Germany).

Burkard, G., Loss, D. \& DiVincenzo, D.P. (1999). Coupled quantum dots as quantum gates, Phys. Rev. B, 59, (2070-2078).

Cantele, G., Ninno, D. \& Iadonisi, G. (2001). Calculation of the Infrared Optical Transitions in Semiconductor Ellipsoidal Quantum Dots, Nano Lett., 1, (121-124).

Cantele, G., Piacente, G., Ninno, D. \& Iadonisi, G. (2002). Optical anisotropy of ellipsoidal quantum dots Phys. Rev., B 66, (113308-1 - 113308-4).

Chestnoy, N., Hull, R. \& Brus, L.,E. (1986). Higher excited electronic states in clusters of ZnSe, CdSe, and ZnS: Spin-orbit, vibronic, and relaxation phenomena, J. Chem. Phys. B 85, (2237-2242).

Chye, Y., White, M.E., Johnston-Halperin, E., Gerardot, B.D., Awschalom D.D. \& Petroff, P.M. (2002). Spin injection from (Ga,Mn)As into InAs quantum dots, Phys. Rev. B, 66, (201301(R)-201304(R)).

Darula, I. \& Barabási, A.,L, (1997). Island Formation and Critical Thickness in Heteroepitaxy Phys. Rev. Lett., 78, (3027-3027).

Daudin, B., Widmann, F., Feuillet, G., Samson, Y., Arlery, M. \& Rouvière, J.L. (1997). Stranski-Krastanov growth mode during the molecular beam epitaxy of highly strained GaN, Phys. Rev. B, 56, (R7069-R7072).

Dawson, P., Göbel, E. O., Pierz, K., Rubel, O., Baranovskii, S. D. \& Thomas, P. (2007). Relaxation and recombination in InAs quantum dots, Phys. Stat. Sol. (b) 244, (2803-2815).

Eaglesham, D.,J. \& Cerullo, M. (1990). Dislocation-free Stranski-Krastanow growth of Ge on Si(100) Phys. Rev. Lett., 64, (1943-1946).

Fessatidis, V., Horing, N. J. M. \& Kashif S. (1999) Retarded Green's function for an electron in a parabolic quantum dot subject to a constant uniform magnetic field and an electric field of arbitrary time dependence and orientation Philosophical Magazine Part B, 79, (77-89).

Foulkes, W.M.C., Mitas, L., Needs, R.J. \& Rajagopal G. (2001). Quantum Monte Carlo simulations of solids. Rev. Mod. Phys., 73, (33-83).

Fujisawa, T., Oosterkamp, T. H., Kouwenhoven, P. \& Wilfred G.(1998). Spontaneous Emission Spectrum in Double Quantum Dot Devices, Science, 282 (932-935).

Fujisawa T., Hayashi T. \& Sasaki S. (2006) Time-dependent single-electron transport through quantum dots, Rep. Prog. Phys., 69 (759-796).

Gershoni, D., Henry, C.,H. \& Baraff, G.,A. (1993) Calculating the optical properties of multidimensional heterostructures: Application to the modeling of quaternary quantum well lasers, IEEE J. Quant. Elec., 29 (2433-2450). 
Ghaisas, S.,V. \& Madhukar, A. (1986). Role of surface molecular reactions in influencing the growth mechanism and the nature of nonequilibrium surfaces: a Monte Carlo study of molecular-beam epitaxy, Phys. Rev. Lett., 56, (1066-2078).

Gross, E., K., U., Runge, E. \& Heinonen, O. (1991). Many-Particle Theory (Taylor \& Francis).

Hayashi, T., Fujisawa, T., Cheong, H.D., Jeong, Y.H. \& Y. Hirayama (2004). Coherent Charge Oscillation in a Semiconductor Double Quantum Dot, IEEE Transactions on Nanotechnology, 3 (300-303).

Hohenberg, P. \& Kohn, W. (1964). Inhomogeneous Electron Gas, Phys. Rev., 136, (864-871).

$\mathrm{Hu}$, X. \& Sarma,S., Das (2000). Hilbert-space structure of a solid-state quantum computer: Two-electron states of a double-quantum-dot artificial molecule, Phys. Rev. A 61, (062301, 062319).

$\mathrm{Hu}$, X. \& Sarma,S., Das (2001). Spin-based quantum computation in multielectron quantum dots, Phys. Rev. A 64, (042312, 042316).

Imamoglu, A., Awschalom, D.D., Burkard, G., DiVincenzo, D.P. Loss, D., Sherwin, M. \& Small, A. (1999). Quantum Information Processing Using Quantum Dot Spins and Cavity QED, Phys. Rev. Lett., 83, (4204-4207).

Jackson, J.D. (1998). Classical Electrodynamics, (Wiley, 3 edition).

Kew, J., Wilby, M.,R. \& Vvedensky, D.,D. (1993). Continuous-space Monte Carlo simulations of epitaxial growth., J. Cryst. Growth, 127, 508.

Khaetskii, A.V. \& Nazarov, Y.V. (2001). Spin-flip transitions between Zeeman sublevels in semiconductor quantum dots, Phys. Rev. B, 64, (125316-125321).

Khor, K.,E. \& Sarma, S.,D. (2000). Quantum dot self-assembly in growth of strainedlayer thin films: a kinetic Monte Carlo study. Phys. Rev. B., 62, (16657-16664).

Kohn, W. \& Sham, L.J. (1965). Self-Consistent Equations Including Exchange and Correlation Effects, Phys. Rev., 140, (A1133-A1138).

Lam, C.,H., Lee, C.,K. \& Sander, L.,M. (2002). Competing roughening mechanisms in strained heteroepitaxy: A fast kinetic Monte Carlo study, Phys. Rev. Lett. 89, (216102-216105).

Lebens, J.,A., Tsai, C.,S., Vahala, K.,J. \& Kuech, T.,F. (1990). Application of selective epitaxy to fabrication of nanometer scale wire and dot structures, Appl. Phys. Lett. 56, (2642, 2644).

Lee, S., Daruka, I., Kim, C.,S., Barabási, A.,L,, Merz, J.,L. \& Furdyna, J.,K. (1998). Dynamics of Ripening of Self-Assembled II-VI Semiconductor Quantum Dots Phys. Rev. Lett, 81, (3479-3482).

Lee, S., Lazarenkova, O.L., Allmen, P, Oyafuso, F. \& Klimeck, G. (2004). Effect of wetting layers on the strain and electronic structure of InAs self-assembled quantum dots, Phys. Rev. $B, 70,(125307-125313)$.

Madhukar, M. (1983) Far from equilibrium vapor phase growth of lattice matched IIIV compound semiconductor interfaces: some basic concepts and Monte-Carlo computer simulations, Surf. Sci., 132, 344-374.

Mohanty, P. (2000). Notes on decoherence at absolute zero, Physica B. 280, (446-452) (and references therein).

Much, F., Ahr, M., Biehl, M. \& Kinzel, W. (2001). Kinetic Monte Carlo simulations of dislocations in heteroepitaxial growth, Europhys. Lett., 56,(791-796).

Nielsen, M. and Chuang, I.L. (2000). Quantum Computation and Quantum Information (Cambridge University Press, Cambridge,).

Orr, B.,G., Kessler, D., Snyder, C.,W. \& Sander, L. M. (1992). A model for strain-induced roughening and coherent island growth, Europhys. Lett., 19,(33). 
Owen, G. (1985). Electron lithography for the fabrication of microelectronic devices , Rep. Prog. Phys. 48, 795.

Pryor, C., Pistol, M.E. \& Samuelson, L. (1997). Electronic structure of strained InP / Ga $a_{0.51} I n_{0.49} P$ quantum dots, Phys. Rev. B 56, (10404-10411).

Pryor, C. (1998). Eight-band calculations of strained InAs/GaAs quantum dots compared with one-, four-, and six-band approximations, Phys. Rev. B 57, (7190-7195).

Pryor, C.E., \& Flatté, M.E. (2003). Accuracy of Circular Polarization as a Measure of Spin Polarization in Quantum Dot Qubits, Phys. Rev. Lett. 91, (257901-257904).

Räsänen, E., Saarikoski, H., Stavrou, V. N., Harju, A., Puska, M. J. \& Nieminen, R. M. (2003). Electronic structure of rectangular quantum dots, Phys. Rev. B 67, (235307-1, 045320-8).

Rastelli, A., Stoffel, M., Tersoff, J., Kar, G.,S. \& Schmidt, O.,G. (2005) Kinetic Evolution and Equilibrium Morphology of Strained Islands, Phys. Rev. Lett. 95, (026103-026106).

Saada, A.,S. (1989). Elasticity:Theory and applications ( R. E. Krieger Publishing Co.).

Sadiku, M.,N.,O. (2001). Numerical Techniques in Electromagnetics ( CRC Press).

Sham, L.J. \& Kohn, W. (1966). One-Particle Properties of an Inhomogeneous Interacting Electron Gas, Phys. Rev., 145, (561-567).

Simserides, C. D., Hohenester, U., Goldoni, G. \& Molinari, E. (2000). Local absorption spectra of artificial atoms and molecules. Phys. Rev. B 62, (13657-13666).

Simserides, C., Zora, A. \& Triberis, G. (2006). Near-field magnetoabsorption of quantum dots. Phys. Rev. B 73, (155313-1, 155313-13).

Simserides, C., Zora, A. \& Triberis, G. (2006). Magneto-Optics of Quantum Dots in the Near Field. International Journal of Modern Physics B 21, (1649-1653).

Sitter, H. (1995). MBE growth mechanisms; studies by Monte-Carlo simulation, Thin Solid Films 267, 37.

Stano, P. \& Fabian, J. (2005). Spin-orbit effects in single-electron states in coupled quantum dots. Phys. Rev. B 72, (155410-1, 155410-14).

Stano, P. \& Fabian, J. (2006). Orbital and spin relaxation in single and coupled quantum dots. Phys. Rev. B 74, (045320-1, 045320-12).

Stavrou, V.N. \& Hu, X. (2005). Charge decoherence in laterally coupled quantum dots due to electron-phonon interactions. Phys. Rev. B 72, (075362-1, 075362-8).

Stavrou, V.N. \& Hu, X. (2006). Electron relaxation in a double quantum dot through two-phonon processes. Phys. Rev. B 73, (205313-1, 205313-5).

Stavrou, V.N. (2007) Suppression of electron relaxation and dephasing rates in quantum dots caused by external magnetic fields. J. Phys.: Condens. Matter 19, (186224-1, 186224-9).

Stavrou, V.N. (2008). Circularly polarized light in coupled quantum dots. J. Phys.: Condens. Matter 20, (395222-1, 395222-4).

Stavrou, V.N. (2009). Polarized light in quantum dot qubit under an applied external magnetic field. Phys. Rev. B 80, (153308-1, 153308-4).

Temkin, H., Dolan, G.,J., Panish, M.,B. \& Chu, S.,N.,G. (1987). Low-temperature photoluminescence from InGaAs/InP quantum wires and boxes, Appl. Phys. Lett. 50, (413-415).

Tersoff, J. (1995). Enhanced Solubility of Impurities and Enhanced Diffusion near Crystal Surfaces. Phys. Rev. Lett. 74, (5080-5083).

Tersoff, J. (1998). Enhanced Nucleation and Enrichment of Strained-Alloy Quantum Dots. Phys. Rev. Lett. 81, (3183-3186).

Thijssen, J.,M. (1998). Computational Physics (Cambridge University Press). 
Thorwart, M., Eckel, J. \& Mucciolo, E.R. (2005). Non-Markovian dynamics of double quantum dot charge qubits due to acoustic phonons. Phys. Rev. B 72, (235320-1, 235320-6).

Tu, Y., \& Tersoff, J. (2007). Coarsening, Mixing, and Motion: The Complex Evolution of Epitaxial Islands Phys. Rev. Lett. 98, (096103-096106).

Varga, Kálmán \& Driscoll, Joseph A. (2011). Computational Nanoscience (Cambridge University Press, Cambridge).

Vurgaftman,I, Meyer, J.R. \& Ram-Mohan, L.R. (2001). Band parameters for III-V compound semiconductors and their alloys. J. A. Appl. Phys. 89, (5815-5875).

Wang,Y. \& Herron, N. (1991) Nanometer-Sized Semiconductor Clusters: Materials Synthesis, Quantum Size Effects, and Photophysical Properties J. Phys. Chem. 95, (525-532).

Witzel, W. M., Hu, X. \& Sarma,S. Das (2007). Decoherence induced by anisotropic hyperfine interaction in Si spin qubits, Phys. Rev. B 76, (035212-1, 035212-8).

Woods, L.M., Reinecke, T.L. \& Lyanda-Geller, Y. (2002). Spin relaxation in quantum dots, Phys. Rev. B 66, (161318-1, 161318-4). 


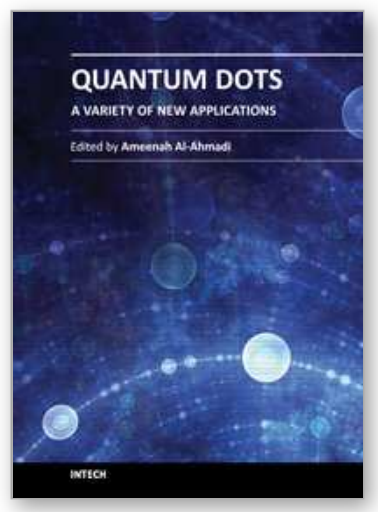

\section{Quantum Dots - A Variety of New Applications}

Edited by Dr. Ameenah Al-Ahmadi

ISBN 978-953-51-0483-4

Hard cover, 280 pages

Publisher InTech

Published online 04, April, 2012

Published in print edition April, 2012

The book "Quantum dots: A variety of a new applications" provides some collections of practical applications of quantum dots. This book is divided into four sections. In section 1 a review of the thermo-optical characterization of CdSe/ZnS core-shell nanocrystal solutions was performed. The Thermal Lens (TL) technique was used, and the thermal self-phase Modulation (TSPM) technique was adopted as the simplest alternative method. Section 2 includes five chapters where novel optical and lasing application are discussed. In section 3 four examples of quantum dot system for different applications in electronics are given. Section 4 provides three examples of using quantum dot system for biological applications. This is a collaborative book sharing and providing fundamental research such as the one conducted in Physics, Chemistry, Biology, Material Science, Medicine with a base text that could serve as a reference in research by presenting up-todate research work on the field of quantum dot systems.

\section{How to reference}

In order to correctly reference this scholarly work, feel free to copy and paste the following:

V. N. Stavrou and G. P. Veropoulos (2012). Spin-Based Quantum Dot Qubits, Quantum Dots - A Variety of New Applications, Dr. Ameenah Al-Ahmadi (Ed.), ISBN: 978-953-51-0483-4, InTech, Available from: http://www.intechopen.com/books/quantum-dots-a-variety-of-new-applications/quantum-dot-qubits

\section{INTECH}

open science | open minds

\author{
InTech Europe \\ University Campus STeP Ri \\ Slavka Krautzeka 83/A \\ 51000 Rijeka, Croatia \\ Phone: +385 (51) 770447 \\ Fax: $+385(51) 686166$ \\ www.intechopen.com
}

\author{
InTech China \\ Unit 405, Office Block, Hotel Equatorial Shanghai \\ No.65, Yan An Road (West), Shanghai, 200040, China \\ 中国上海市延安西路65号上海国际贵都大饭店办公楼 405 单元 \\ Phone: +86-21-62489820 \\ Fax: +86-21-62489821
}


(C) 2012 The Author(s). Licensee IntechOpen. This is an open access article distributed under the terms of the Creative Commons Attribution 3.0 License, which permits unrestricted use, distribution, and reproduction in any medium, provided the original work is properly cited. 